\title{
KCN/11 activating mutations are associated with developmental delay, epilepsy and neonatal diabetes syndrome and other neurological features
}

\author{
Anna L Gloyn*1,2, Catherine Diatloff-Zito ${ }^{3}$, Emma L Edghill ${ }^{2}$, \\ Christine Bellanné-Chantelot ${ }^{4}$, Sylvie Nivot ${ }^{5}$, Régis Coutant ${ }^{6}$, Sian Ellard ${ }^{2}$, \\ Andrew T Hattersley ${ }^{2}$ and Jean Jacques Robert ${ }^{3}$
}

\footnotetext{
${ }^{1}$ Diabetes Research Laboratories, Oxford Centre for Diabetes Endocrinology and Metabolism, University of Oxford, UK; ${ }^{2}$ Institute of Biomedical and Clinical Science, Peninsula Medical School, Exeter, UK; ${ }^{3}$ Federation of Pediatrics, NeckerEnfants Malades University Hospital, Paris, France; ${ }^{4}$ Department of Cytogenetics, Saint-Antoine University Hospital, Paris, France; ${ }^{5}$ Department of Pediatrics, University Hospital, Angers, France; ${ }^{6}$ Department of Pediatric Diabetology, South University Hospital, Rennes, France
}

Heterozygous activating mutations in the gene encoding for the ATP-sensitive potassium channel subunit Kir6.2 (KCNJ11) have recently been shown to be a common cause of permanent neonatal diabetes. Kir6.2 is expressed in muscle, neuron and brain as well as the pancreatic beta-cell, so patients with KCN/11 mutations could have a neurological phenotype in addition to their diabetes. It is proposed that some patients with $K C N 111$ mutations have neurological features that are part of a discrete neurological syndrome termed developmental Delay, Epilepsy and Neonatal Diabetes (DEND), but there are also neurological consequences of chronic or acute diabetes. We identified KCNJ11 mutations in four of 10 probands with permanent neonatal diabetes and one affected parent; this included the novel C166F mutation and the previously described V59M and R201H. Four of the five patients with mutations had neurological features: the patient with the C166F mutation had marked developmental delay, severe generalised epilepsy, hypotonia and muscle weakness; mild developmental delay was present in the patient with the V59M mutation; one patient with the R201H mutation had acute and chronic neurological consequences of cerebral oedema and another had diabetic neuropathy from chronic hyperglycaemia. In conclusion, the clinical features in these patients support the existence of a discrete neurological syndrome with $K C N J 11$ mutations. The severe DEND syndrome was seen with the novel C166F mutation and mild developmental delay with the V59M mutation. These features differ markedly from the neurological consequences of acute or chronic diabetes.

European Journal of Human Genetics (2006) 14, 824-830. doi:10.1038/sj.ejhg.5201629; published online 3 May 2006

Keywords: permanent neonatal diabetes; Kir6.2; KATP channel; DEND syndrome; genetics; KCNJ11

\footnotetext{
*Correspondence: Dr AL Gloyn, Diabetes Research Laboratories, Oxford Centre for Diabetes, Endocrinology and Metabolism, Churchill Hospital, Old Road, Headington, Oxford, UK.

Tel: + 441865 857343; Fax: + 441865857299 ;

E-mail: anna.gloyn@drl.ox.ac.uk

Received 16 November 2005; revised 19 January 2006; accepted 8 February 2006; published online 3 May 2006
}

Introduction

Heterozygous activating mutations in the KCNJ11 gene encoding the Kir6.2 subunit of the pancreatic beta-cell ATP-sensitive potassium ( $\mathrm{K}_{\mathrm{ATP}}$ ) channel have recently been described as a common and important cause of neonatal diabetes. $^{1,2}$ They have been reported to account for 
between 33 and $50 \%$ of cases of permanent neonatal diabetes (PNDM). ${ }^{1,3-5}$ The majority of mutations causing PNDM were shown to arise de novo and common mutation sites were identified at residues 201 and 59. ${ }^{1,3-6}$

Kir6.2 is not just expressed in the pancreatic beta-cell, it is expressed in many other tissues including muscle, neuron and brain; ${ }^{7,8}$ therefore, mutations could result in neurological features. Whereas most patients have isolated neonatal diabetes, some patients with KCNJ11 mutations have developmental delay, muscle weakness and epilepsy as well as diabetes. ${ }^{1}$ This has been proposed to be part of a discrete syndrome associated with specific mutations, termed developmental delay, epilepsy and neonatal diabetes (DEND) syndrome. ${ }^{9}$ However, to date only three patients have been identified with developmental delay and epilepsy, and it remains unclear as to whether the clustering of these features is a chance finding or related to the mutations. ${ }^{1}$

In this study, we report the screening of KCNJ11 in 10 patients with neonatal diabetes and report the identification of four subjects with activating mutations, including a patient with a novel mutation resulting in full DEND syndrome.

\section{Methods}

\section{Subjects studied}

DNA was sequenced from 10 probands with PNDM. Patients were recruited from Hôpital Necker - Enfants Malades and corresponding clinics. None of these patients were included in the previous report of French patients. ${ }^{4}$ Abnormalities of chromosome 6q24, mutations in the glucokinase $(G C K)$ gene and pancreatic agenesis were excluded. Informed consent was obtained from all participants or their parents.

\section{Mutation analysis}

The coding region and the intron-exon boundaries of the ATP-sensitive channel subunit Kir6.2 (KCNJ11) gene were amplified from genomic DNA by PCR as described previously. ${ }^{1}$ The products were sequenced by standard methods on an ABI 3100 (Applied Biosystems, Warrington, UK). Family relationships were confirmed using a panel of 10 microsatellite markers. ${ }^{10} \mathrm{KCNJ} 11$ was also sequenced in 100 nondiabetic individuals.

\section{Results \\ Mutational analyses}

We identified heterozygous missense KCNJ11 mutations in four of the 10 probands with PNDM: the novel C166F and two previously reported heterozygous mutations V59M and R201H. In family NECKER 29, a novel missense mutation resulting in the substitution of cysteine for phenylalanine at codon 166 (C166F) (TGC>TTC) was detected (underlining illustrates the base which has been mutated). In families NECKER 4 and 6, a missense mutation resulting in the substitution of histidine for arginine at codon $201(\mathrm{R} 201 \mathrm{H})(\mathrm{CGT}>\mathrm{CAT})$ was identified in both probands. In family NECKER 6 , the mutation was present in the mother of the proband who also had neonatal diabetes. In family ANGERS 1 , methionine was substituted for valine at residue 59 (V59M) ( $\underline{\text { TG }}>\underline{\mathrm{ATG}}$ ). In all families, diabetes was only seen in subjects with $K C N J 11$ mutations and all subjects without these mutations were nondiabetic. DNA was available from the unaffected parents in all families and the mutations were shown to arise de novo in the oldest affected member in all families with familial transmission to an affected offspring occurring in one family NECKER 6 (Figure 1). None of the mutations were present in 100 nondiabetic UK Caucasian subjects. In addition, several previously recognized polymorphisms (E23K, A190A, L267L, L270V, I337V) were identified. The mutated amino acids are conserved among human, rats, mice and bullfrogs.

\section{Clinical characteristics of subjects with $K C N J I I$ mutations}

The clinical characteristics of the subjects with mutations are summarised in Table 1.

The proband with the C166F mutation (NECKER 29 II:1) was born to nonconsanguineous parents following an uneventful pregnancy and spontaneous term delivery. At 28 weeks, an ultrasound study showed severe intrauterine growth retardation ( $<3$ rd percentile) and her birth weight was only $2465 \mathrm{~g}$ (1.6th percentile). From the first day of life she developed feeding problems owing to poor suckling and failure to thrive. At 6 weeks, head control was lacking, and sucking and crying were poor. At 3 months, diabetes mellitus was diagnosed on the basis of polyuria and polydipsia and confirmed by the finding of marked hyperglycaemia $(27 \mathrm{mmol} / \mathrm{l})$ and ketosis, but not acidosis. Autoantibodies associated with type I diabetes were not detected. Diabetes was controlled with an insulin pump on a dose of $0.1 \mathrm{IU} / \mathrm{kg} /$ day. At 3 months, she developed seizures with repeated episodes of truncal flexion. The details of the seizures have been reported in detail elsewhere. ${ }^{11}$ The seizures were associated with an abnormal EEG, which shows hypsarrhythmia (N Bahi-Busson, M Eisermann, S Nivot, C Bellane-Chantelot, P Dulac, N Bach, P Plouin, P De Lonlay, personal communication). Progressive neurological deterioration resulted in social withdrawal and poor eye contact. She was noted to have mild dysmorphic features; a prominent metopic suture, down turned mouth and bilateral ptosis (Figure 2). Examination revealed diffuse hypotonia, with poor head and trunk support, poor eye contact and weak tendon reflexes and without muscular atrophy. The proband died from aspiration pneumonia at the age of 6 months.

The proband from family NECKER 4 with the R201H mutation was of Algerian descent and born to nonconsan- 

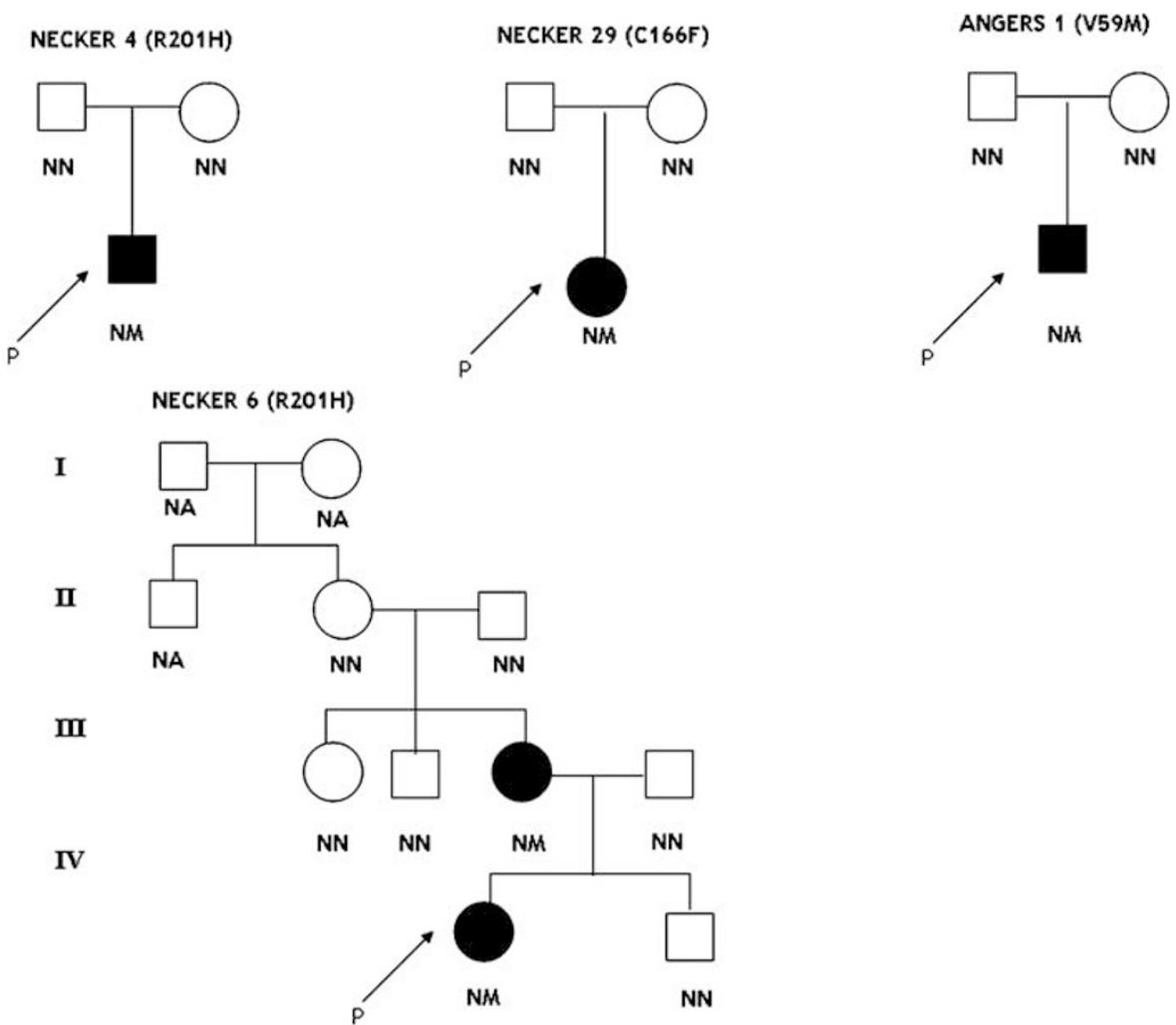

Figure 1 Pedigrees of families with KCNJ11 mutations. Diabetes status and mutations in the gene encoding Kir6.2 in four families. These partial pedigrees show families with the V59M, C166F and R201H mutations. In all pedigrees for which parental DNA was available, family relationships were confirmed by a panel of 10 microsatellites. In NECKER 4, NECKER 29 and ANGERS 1, spontaneous mutations explain the absence of permanent neonatal diabetes in the parents and its presence in a child. Squares represent male family members, circles female family members and diamonds sex not defined. Black circles and squares represent persons with neonatal diabetes. A two-letter code for allele status is shown underneath each symbol: $\mathrm{N}$, no mutation; $\mathrm{M}$, mutation; and NA, not available for testing. $\mathrm{P}$ and an arrow denote the proband in each family.

guineous parents. He was born at 38 weeks with a birth weight of $2615 \mathrm{~g}$ (12th percentile). At the age of 4 weeks, the child presented with tachypnoea, dehydration and diabetic ketoacidosis (blood glucose of $27 \mathrm{mmol} / \mathrm{l}$, ketonuria and a $\mathrm{pH}$ of 6.95). He was admitted to the intensive care unit where he received intravenous (i.v.) fluid and i.v. insulin. At 3 days after diagnosis of diabetes, he developed reduced consciousness and generalised seizures, which were considered to be owing to cerebral oedema. Following this, he showed axial hypotonia and had developmental delay, not walking until 3 years of age. The child had possible mild dysmorphic features: with a small short nose, long philtrum, thick ears and clinodactyly of the last four fingers. Autoantibodies associated with type I diabetes were not detected. The proband had a normal high-resolution karyotype.

The proband from family ANGERS 1 had the V59M mutation. They were born at term with a birth weight of $2980 \mathrm{~g}$ (11th percentile) to nonconsanguineous Algerian parents. At the age of 1 month and a half, he presented with polyuria-polydipsia, vomiting, tachypnoea and dia- betic ketoacidosis, and was diagnosed on the basis of a blood glucose of $44 \mathrm{mmol} / \mathrm{l}$, ketonuria and a $\mathrm{pH}$ of 6.99 . He was treated with normal saline and insulin $0.5-0.6 \mathrm{IU} / \mathrm{kg} /$ day. Autoantibodies associated with type I diabetes were not detected, C-peptide was $<0.2 \mathrm{ng} / \mathrm{ml}$ and HbA1c was $8.2 \%$. There was evidence of mild motor developmental delay. At the age of 8 months, he showed mild axial tone and was unable to sit up unaided. He could sit unaided by 10 months, by 13 months he was able to stand and he walked at 18 months.

The proband (IV:1) and her mother (III:3) from family NECKER 6 both had the R201H mutation. The proband was born at 38.5 weeks after an uneventful pregnancy under strict supervision because of the mother's diabetes, which was managed by using an insulin pump. Her birth weight was $2730 \mathrm{~g}$ (19th percentile). Hyperglycaemia was discovered on the first day of life $(9.6$ and $11.4 \mathrm{mmol} / \mathrm{l})$ but was not treated initially. At 17 days after birth, blood glucose was still $>15 \mathrm{mmol} / \mathrm{l}$ and insulin treatment was initiated at $0.5-0.6 \mathrm{IU} / \mathrm{kg} /$ day. Autoantibodies associated with type I diabetes were not detected; fasting C-peptide was 
Table 1 Clinical characteristics of patients with KCNJ11 mutations

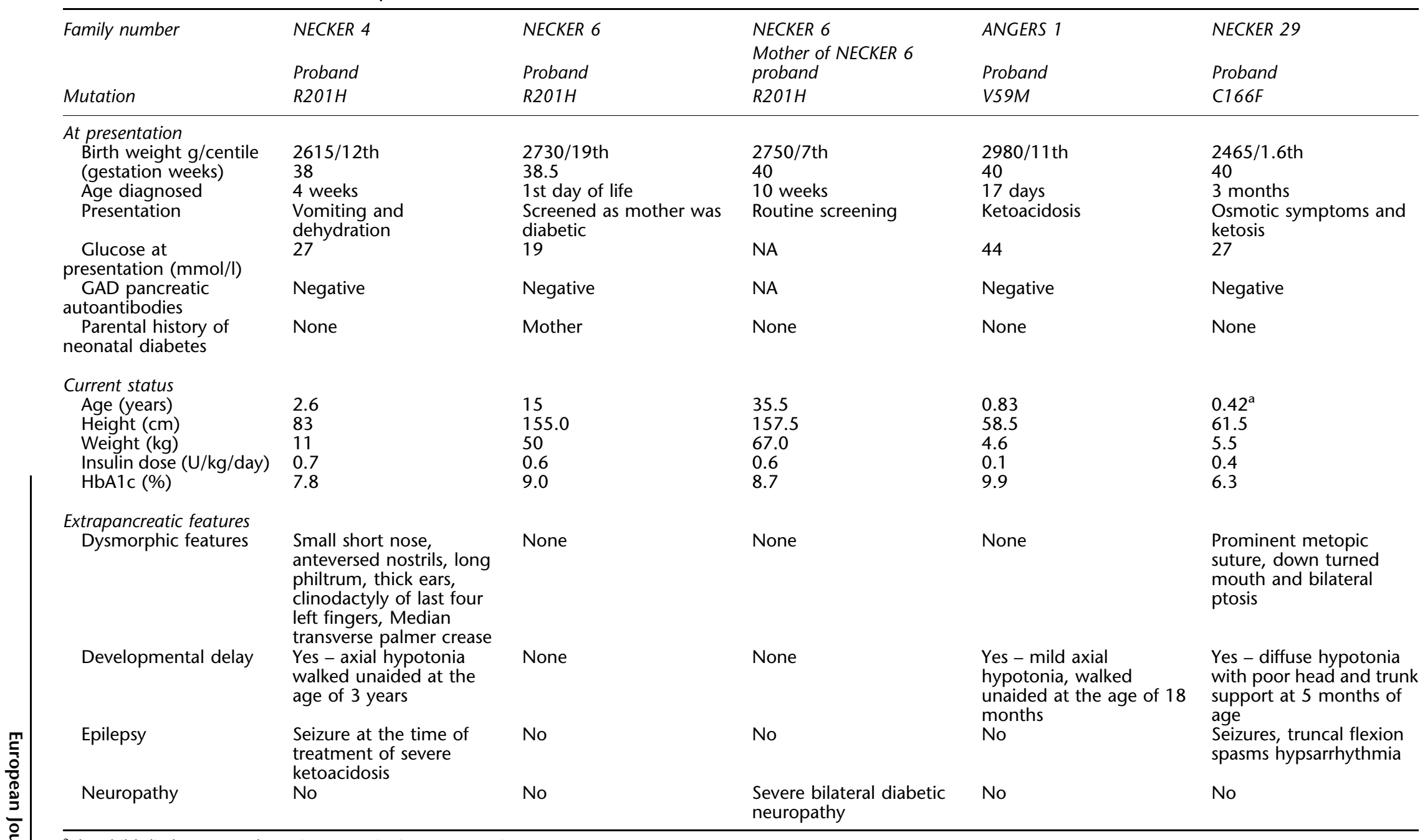

${ }^{a}$ The child died at 6 months owing to aspiration pneumonia. NA, data not available. 


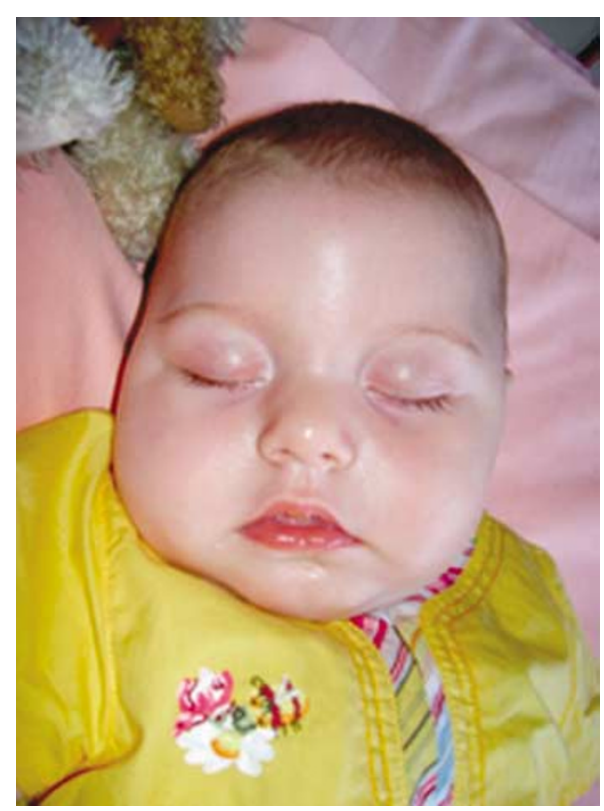

Figure 2 Photograph of NECKER 29.

$0.06 \mathrm{mmol} / \mathrm{l}$ and only $0.18 \mathrm{mmol} / \mathrm{l}$ after IV glucagon. At the age of 16 years, insulin dose was $0.6 \mathrm{IU} / \mathrm{kg} / \mathrm{day}$, and HbA1c 9.5 and development was normal.

The proband's mother (III:3) was born at term with a birth weight of $2750 \mathrm{~g}$ ( 7 th percentile). She had recurrent ear infections and was discovered, on systematic hospital screening for condition, to have hyperglycaemia. Glycaemia was managed with dietary advice only but did not remit. Insulin treatment started at 4 years of age using a single daily injection as glucose values were still consistently in double figures. She had normal psychomotor development. As an adult, she developed bilateral nonproliferative diabetic retinopathy and bilateral diabetic peripheral neuropathy, which lead to diabetic foot ulceration and at the age of 36 years, the amputation of her left foot. At 36 years, her insulin dose is $0.4 \mathrm{IU} / \mathrm{kg} /$ day and HbA1c 8.7\%.

\section{Discussion}

We have identified heterozygous mutations in the KCNJ11 gene in four of 10 probands with neonatal diabetes. In one family, the mother of the proband also has diabetes as a result of the KCNJ11 mutation and four patients have the previously reported mutations $\mathrm{R} 201 \mathrm{H}$ (two probands and one parent) and V59M. Four of the five patients with KCNJ11 mutations have neurological features and these represent different aspects of neurological dysfunction associated with KCNJ11 mutations.

The prevalence of KCNJ11 mutations in our cohort of $40 \%$ is consistent with KCNJ11 mutations being a common cause of neonatal diabetes. In other large series of neonatal diabetes, the prevalence ranges between 33 and 50\%. ${ }^{1,3-5}$ The patients in this series do not include those in the previous study, ${ }^{4}$ giving an overall prevalence of KCNJ11 mutations in France of $13 / 26=50 \%$.

The diabetes in the five patients with KCNJ11 mutations are generally similar to that seen in other patients with KCNJ11 mutations. ${ }^{12}$ They show low birth weight consistent with low insulin production in utero and present with marked hyperglycaemia and ketoacidosis (two patients). The exception is the mother (III:III) in family NECKER 6 who was detected as having hyperglycaemia at 2 months of age but was not managed with insulin until she was 4 years old. This is exceptional as most patients with the $\mathrm{R} 201 \mathrm{H}$ mutation produce very little insulin (as shown by Cpeptide $<0.20 \mathrm{mmol} / \mathrm{l}$ even when stimulated by glycaemia or glucagon). ${ }^{1,3}$ Transient neonatal diabetes has been described in three patients with the G53S, G53R and I182V KCNI11 mutations. ${ }^{2}$ Functional studies have suggested that a characteristic of the mutations that cause TNDM was that their response to ATP was more similar to wild-type than the R201C mutation normally associated with isolated PNDM. The finding of a child who can go for 4 years without insulin suggests that for a given mutation there is variation in the severity of phenotype. Patients with endogenous insulin secretion despite the R201H mutation have been described in two other patients with the $\mathrm{R} 201 \mathrm{H}$ mutation. ${ }^{13,14}$ This variability in phenotype between subjects with the same mutation suggests that other factors such as genetic background and the environment play a role in modifying the phenotype.

We have described a patient with the novel mutation C166F that has all the features of the DEND syndrome with developmental delay, epilepsy and neonatal diabetes. This is only the fourth patient to be described with generalised epilepsy before 12 months and a KCNJ11 mutation. The clinical features are extremely similar to the three cases described in the initial paper. ${ }^{1}$ Hypsarrhythmia has been described before $^{1}$ and there is striking similarity of appearance with mild dysmorphic features that include the prominent metopic suture, bilateral ptosis and down turned mouth. This strongly supports that DEND syndrome is likely to be a direct result of a severe activating mutation in KCNJ11, which alters the function of the channel in the brain, muscle and nerves in addition to the pancreatic beta-cell. The diabetes of the patients with DEND syndrome is similar to that found in patients without neurological features and is no more severe than those without neurological features. ${ }^{1}$ The aspiration pneumonia that occurred in this patient is likely to have been at least in part owing to DEND syndrome with the marked muscular weakness this child had, reducing the ability to cough. This is the second reported case where the child is thought to have died before the age of 3 years $^{1}$ with aspiration pneumonia and the risk of this complication is an important clinical consideration. 
The functional characterisation of the mutations associated with DEND syndrome support the hypothesis of more functionally severe mutations resulting in the syndrome. Functional studies have demonstrated that the mutations identified in patients with syndromic PNDM (V59G, G52R, I296L) result in a larger $\mathrm{K}_{\mathrm{ATP}}$ current and greater change in ATP sensitivity than those which cause PNDM alone (R201C), suggesting that larger $\mathrm{K}_{\mathrm{ATP}}$ currents are required to influence extrapancreatic cell function. ${ }^{9,15}$ Residue C166 has been extensively mutated ${ }^{16,17}$ and functional studies have shown that the C166F mutation results in a channel with a marked increase in open probability (0.77 vs 0.11$)$ and a reduced ATP sensitivity $\left(\mathrm{Ki}_{\mathrm{ATP}}, 5.3 \mathrm{mM} v s\right.$ wild type $\left.175 \mu \mathrm{M}\right)$ where $\mathrm{Ki}_{\mathrm{ATP}}$ is the ATP concentration at which inhibition is half-maximal. ${ }^{17}$ The fact that the channel open probability is increased implies a role for this residue in channel gating. ${ }^{17}$ This would fit with the observation that mutations which cause PNDM alone predominantly impair ATP sensitivity directly (at the binding site), whereas those associated with severe disease act indirectly by biasing the channel conformation towards the open state. ${ }^{9}$

We also describe a patient with the V59M mutation with only moderate developmental delay and no epilepsy in addition to their diabetes. This cluster of features has been termed the intermediate DEND syndrome, iDEND. ${ }^{12}$ This mutation is very strongly related to iDEND: nine of the 12 cases with this mutation reported to date have developmental delay in addition to their diabetes. ${ }^{1,3-5,12}$ In this case, the neurological features were subtle with axial hypotonia and delayed motor milestones. This suggests that even with a single mutation, there is a considerable variation in the severity of the developmental delay and muscle weakness. The functional characterisation of the V59M mutation has shown that the severity of this mutation is intermediate between mutations usually associated with isolated diabetes alone and those associated with the DEND phenotype. ${ }^{18}$

In addition to the neurological features arising from the KCNJ11 mutations, we also show that neurological features can arise from chronic or acute hyperglycaemia. The mother of NECKER 6 has a severe bilateral diabetic neuropathy affecting both legs, which ultimately resulted in diabetic ulceration and amputation. This is the first description of neurological complications from chronic hyperglycaemia in a patient with KCNJ11 diabetes. The HbA1c value of $8.7 \%$ suggests that her current control is comparable with the patients previously reported (7.4$10 \%$ ), and it is likely that her poor control (HbA1c values were as high as 13\%) in adolescence and early adulthood could have contributed to her early and severe complications. The proband from NECKER 4 had acute neurological complications of diabetes and its treatment, as they presented with seizures from cerebral oedema following treatment of ketoacidosis. On the basis of clinical and neuroimaging findings, this was thought to represent acute cerebral oedema, which is a known complication of severe ketoacidosis in young children. ${ }^{19}$ His mild dysmorphic features are interesting, but they are unlike the classical features reported in the other cases of syndromic PNDM. ${ }^{1}$ The dysmorphism is unexplained as he has a normal karyotype and is from a nonconsanguineous family.

The identification of a KCNJ11 mutation has implications for the clinical management of these patients. Patients may be treated successfully with oral sulphonylureas rather than insulin, but there is no evidence at present that it will improve neurological function. $3,13,20-22$ Two patients (NECKER 6 IV:1, III:3) have successfully been transferred from insulin to sulphonylureas. Clearly, neurological problems that are not associated with the KCNJ11 mutation but rather as a complication of the diabetes or an unrelated condition could not be altered by sulphonylureas closing the $\mathrm{K}_{\mathrm{ATP}}$ channel. Whether the severe functional defect associated with DEND syndrome will be helped by sulphonylurea therapy is uncertain, especially as in in vitro the response is limited with these mutations. ${ }^{9}$

The possible causes of neurological dysfunction in patients with KCNJ11 mutations include (i) a direct effect of the mutated channel altering channel function in muscle, axon and cortex, (ii) an acute consequence of the neonatal diabetes as either severe hypoglycaemia (ketoacidosis) or very severe hyperglycaemia, (iii) the long-term impact of diabetes either diabetes-specific complications or repeated severe hypoglycaemia or (iv) incidental neurological features unrelated to either the Kir6.2 channel subunit or diabetes. The patients here show the spectrum of aetiologies.

In conclusion, we have identified four probands with mutations in the KCNJ11 gene in a cohort of 10 patients with permanent neonatal diabetes. Four of the five patients with mutations have neurological features, which represent the range of neurological deficits seen in this condition. The effect of a mutated $\mathrm{K}_{\mathrm{ATP}}$ channel in neurological tissues and muscle is further confirmed by the finding of a additional case of DEND syndrome in a patient with the novel $\mathrm{C} 166 \mathrm{~F}$ mutation and the mild developmental delay seen in the patient with the V59M mutation. However, we also give evidence that care needs to be taken when predicting the phenotype for a specific mutation. Patients can also present with neurological features that are probably due to the effect of acute and chronic diabetes. In patients with KCNJ11 mutations, the majority of the neurological features result from the effect of a mutated Kir6.2 protein in muscle, nerve and brain.

\section{Acknowledgements}

This work was funded in Exeter by the Wellcome Trust. This work was funded in France by the Association Aide aux Jeunes Diabetiques and 
Centre National de la Recherche Scientifique (CNRS). ALG is a Diabetes UK RD Lawrence Research Fellow, and ATH is a Wellcome Trust Clinical Research Leave Fellow. We thank Dr Jean-Pierre Brossier (La Roche sur Yon, France) who referred patient ANGERS 1 and Drs Helen Stewart and Karen Temple for their clinical expertise and $\mathrm{Dr}$ Beverley Shield for her statistical expertise.

\section{References}

1 Gloyn AL, Pearson ER, Antcliff JF et al: Activating mutations in the gene encoding the ATP-sensitive potassium-channel subunit Kir6.2 and permanent neonatal diabetes. N Engl J Med 2004; 350: $1838-1849$.

2 Gloyn AL, Reimann F, Girard C et al: Relapsing diabetes can result from moderately activating mutations in KCNJ11. Hum Mol Genet 2005; 14: 925-934.

3 Sagen JV, Raeder H, Hathout E et al: Permanent neonatal diabetes due to mutations in KCNJ11 encoding Kir6.2: patient characteristics and initial response to sulfonylurea therapy. Diabetes 2004; 53: $2713-2718$.

4 Vaxillaire M, Populaire C, Buisiah K et al: Kir6.2 mutations are a common cause of permanent neonatal diabetes in a large cohort of French patients. Diabetes 2004; 53: 2719-2722.

5 Massa O, Iafusco D, D'Amato E et al: KCNJ11 activating mutations in Italian patients with permanent neonatal diabetes. Hum Mutat 2004; 25: 22-27.

6 Gloyn AL, Cummings EA, Edghill EL et al: Permanent neonatal diabetes due to paternal germline mosaicism for an activating mutation of the KCNJ11 Gene encoding the Kir6.2 subunit of the beta-cell potassium adenosine triphosphate channel. I Clin Endocrinol Metab 2004; 89: 3932-3935.

7 Inagaki N, Gonoi T, Clement JP et al: Reconstitution of $\mathrm{I}_{\mathrm{KATP}}$ : an inward rectifier subunit plus the sulfonylurea receptor. Science 1995; 270: 1166-1170.

8 Karschin C, Ecke C, Ashcroft FM, Karchin A: Overlapping distribution of K (ATP) channel-forming Kir6.2 subunit and the sulphonylurea receptor SUR1 in rodent brain. FEBS Lett 1996; 401: 59-64.

9 Proks P, Antcliff JF, Lippiat J, Gloyn AL, Hattersley AT, Ashcroft FM: Molecular basis of Kir6.2 mutations associated with neonatal diabetes or neonatal diabetes plus neurological features. Proc Natl Acad Sci USA 2004; 101: 17539-17544.

10 Frayling TM, McCarthy MI, Walker M et al: No evidence for linkage at candidate type 2 diabetes susceptibility loci on chromosomes 12 and 20 in United Kingdom Caucasians. J Clin Endocrinol Metab 2000; 85: 853-857.

11 Bahi-Busson N, Bellane-Chantelot C, Eisermann M et al: Early epileptic encephalopathy and neonatal diabetes associated with mutations in the ATP sensitive potassium channel. Am J Hum Genet 2004; 75 (Suppl 1): 323.

12 Hattersley AT, Ashcroft FM: Activating mutations in kir6.2 and neonatal diabetes: new clinical syndromes, new scientific insights, and new therapy. Diabetes 2005; 54: 2503-2513.

13 Klupa T, Edghill EL, Nazim J et al: The identification of a R201H mutation in KCNJ11, which encodes Kir6.2, and successful transfer to sustained-release sulphonylurea therapy in a subject with neonatal diabetes: evidence for heterogeneity of beta cell function among carriers of the $\mathrm{R} 201 \mathrm{H}$ mutation. Diabetologia 2005; 48: 1029-1031.

14 Colombo C, Delvecchio M, Zecchino C, Faienza MF, Cavallo L, Barbetti F: Transient neonatal diabetes mellitus is associated with a recurrent (R201H) KCNJ11 (KIR6.2) mutation. Diabetologia 2005; 48: 2439-2441.

15 Proks P, Girard C, Haider S et al: A gating mutation at the internal mouth of the Kir6.2 pore is associated with DEND syndrome. EMBO Rep 2005; 6: 470-475.

16 Piao $\mathrm{H}$, Cui $\mathrm{N}, \mathrm{Xu} \mathrm{H}$ et al: Requirement of multiple protein domains and residues for gating K(ATP) channels by intracellular pH. J Biol Chem 2001; 276: 36673-36680.

17 Trapp S, Proks P, Tucker SJ, Ashcroft FM: Molecular analysis of ATP-sensitive $\mathrm{K}$ channel gating and implications for channel inhibition by ATP. J Gen Physiol 1998; 112: 333-349.

18 Proks P, Girard C, Ashcroft FM: Functional effects of KCNJ11 mutations causing neonatal diabetes: enhanced activation by MgATP. Hum Mol Genet 2005; 14: 2717-2726.

19 Edge JA, Hawkins MM, Winter DL, Dunger DB: The risk and outcome of cerebral oedema developing during diabetic ketoacidosis. Arch Dis Child 2001; 85: 16-22.

20 Codner E, Flanagan S, Ellard S, Garcia H, Hattersley AT: High-dose glibenclamide can replace insulin therapy despite transitory diarrhoea in early-onset diabetes caused by a novel R201L Kir6.2 mutation. Diabetes Care 2005; 28: 758-759.

21 Zung A, Glaser B, Nimri R, Zadik Z: Glibenclamide treatment in permanent neonatal diabetes mellitus due to an activating mutation in Kir6.2. J Clin Endocrinol Metab 2004; 89: 5504-5507.

22 Yorifuji T, Nagashima K, Kurokawa K et al: The C42R mutation in the Kir6.2 (KCNJ11) gene as a cause of transient neonatal diabetes, childhood diabetes, or later-onset, apparently type 2 diabetes mellitus. J Clin Endocrinol Metab 2005; 90: 3174-3178. 